\title{
Liquid-vapour equilibria of the methyl esters of alkanoic acids: vapour pressures as a function of temperature and standard thermodynamic function changes
}

\author{
Aad C.G. van Genderen, J. Cees van Miltenburg, Jacobus G. Blok, \\ Mark J. van Bommel, Paul J. van Ekeren, Gerrit J.K. van den Berg, Harry A.J. Oonk* \\ Chemical Thermodynamics Group, Utrecht University, Padualaan 8, 3584 CH Utrecht, The Netherlands
}

Received 21 December 2001; accepted 19 April 2002

\begin{abstract}
The vapour pressures as a function of temperature of the methyl esters of alkanoic acids from butanoic to eicosanoic acid, have been measured using two different techniques: the static diaphragm and spinning rotor manometry and the combined torsion mass-loss effusion. Combined with data available from literature, the new data have been processed using the thermodynamic regression equation proposed by Clarke and Glew. The results of this research are reliable vapour pressures and the thermodynamic properties of vaporisation.
\end{abstract}

(c) 2002 Elsevier Science B.V. All rights reserved.

Keywords: Data; Vapour pressure; Vapour-liquid equilibria; Enthalpy; Gibbs energy; Methyl esters of alkanoic acids

\section{Introduction}

In our laboratory, the vapour pressures have been measured of a number of methyl and ethyl esters of alkanoic acids. The experimental details and the results of the investigation were presented by van Bommel in his thesis [1]. In order to make van Bommel's data more accessible, we have opted for an approach in which the data are combined with data available from other sources to arrive at critically assessed sets of vapour pressure, valid over a large range of temperature.

In this communication, we present experimental data and the results of our assessment on the methyl esters of alkanoic acids, from butanoic to eicosanoic acid.

For the mathematical processing of the data, we use the three-parameter form of the thermodynamic regression equation introduced by Clarke and Glew [2].

$$
R \ln \left(\frac{p}{p^{0}}\right)=\frac{-\Delta_{\text {vap }} G_{\mathrm{m}}^{\circ}(\theta)}{\theta}+\Delta_{\text {vap }} H_{\mathrm{m}}^{\circ}(\theta)\left\{\frac{1}{\theta}-\frac{1}{T}\right\}+\Delta_{\text {vap }} C_{p, \mathrm{~m}}^{\circ}\left\{\frac{\theta}{T}-1+\ln \left(\frac{T}{\theta}\right)\right\}
$$

* Corresponding author. Tel.: +31-30-2533506; fax: +31-30-2533946.

E-mail address: oonk@chem.uu.nl (H.A.J. Oonk). 
Table 1

Vapour pressure data [1], and static diaphragm manometry

\begin{tabular}{|c|c|c|c|c|c|c|c|}
\hline$T(\mathrm{~K})$ & $p(\mathrm{~Pa})$ & $T(\mathrm{~K})$ & $p(\mathrm{~Pa})$ & $T(\mathrm{~K})$ & $p(\mathrm{~Pa})$ & $T(\mathrm{~K})$ & $p(\mathrm{~Pa})$ \\
\hline \multicolumn{8}{|c|}{ Methyl butanoate } \\
\hline 293.06 & 3216 & 300.78 & 4911 & 307.23 & 6766 & 313.34 & 9111 \\
\hline 295.67 & 3711 & 300.97 & 4924 & 309.32 & 7500 & & \\
\hline 298.25 & 4282 & 305.63 & 6253 & 311.15 & 8199 & & \\
\hline \multicolumn{8}{|c|}{ Methyl pentanoate } \\
\hline 293.34 & 1044 & 308.05 & 2446 & 318.03 & 4134 & 328.02 & 6726 \\
\hline 297.75 & 1361 & 312.90 & 3174 & 322.47 & 5155 & & \\
\hline \multicolumn{8}{|c|}{ Methyl hexanoate } \\
\hline 297.95 & 451.2 & 308.13 & 875.5 & 318.25 & 1545 & 328.05 & 2631 \\
\hline 302.91 & 620.5 & 312.93 & 1139 & 323.02 & 2003 & 333.00 & 3356 \\
\hline \multicolumn{8}{|c|}{ Methyl heptanoate } \\
\hline 310.60 & 353.1 & 316.75 & 521.5 & 324.75 & 838.4 & 334.79 & 1474 \\
\hline 311.50 & 374.3 & 320.89 & 669.4 & 329.71 & 1113 & 340.35 & 1982 \\
\hline \multicolumn{8}{|c|}{ Methyl octanoate } \\
\hline 312.98 & 149.5 & 322.60 & 282.4 & 332.95 & 533.5 & 348.42 & 127.1 \\
\hline 317.86 & 207.3 & 327.91 & 394.0 & 337.93 & 714.0 & & \\
\hline \multicolumn{8}{|c|}{ Methyl nonanoate } \\
\hline 308.71 & 39.72 & 328.28 & 157.0 & 345.97 & 465.8 & 350.03 & 588.9 \\
\hline 312.45 & 52.75 & 333.21 & 214.0 & 357.68 & 714.6 & 353.40 & 894.7 \\
\hline 316.38 & 70.46 & 336.60 & 263.9 & & & & \\
\hline \multicolumn{8}{|c|}{ Methyl decanoate } \\
\hline 310.03 & 16.13 & 321.56 & 39.15 & 333.23 & 90.2 & 348.19 & 228.9 \\
\hline 313.75 & 21.57 & 325.48 & 52.18 & 338.32 & 126.5 & 351.31 & 277.6 \\
\hline 317.65 & 29.17 & 329.75 & 70.80 & 343.64 & 171.8 & & \\
\hline \multicolumn{8}{|c|}{ Methyl undecanoate } \\
\hline 331.81 & 32.80 & 337.66 & 49.89 & 344.52 & 78.80 & 351.21 & 122.3 \\
\hline 334.60 & 41.21 & 340.71 & 61.26 & 347.60 & 97.17 & & \\
\hline \multicolumn{8}{|c|}{ Methyl dodecanoate } \\
\hline 332.38 & 13.97 & 338.35 & 21.89 & 346.01 & 38.66 & 350.24 & 51.95 \\
\hline 335.33 & 17.54 & 340.25 & 25.30 & 348.06 & 44.47 & 357.29 & 84.76 \\
\hline \multicolumn{8}{|c|}{ Methyl tridecanoate } \\
\hline 338.54 & 9.439 & 344.30 & 14.91 & 351.43 & 24.72 & & \\
\hline 341.31 & 12.01 & 347.92 & 19.19 & 355.31 & 32.44 & & \\
\hline \multicolumn{8}{|c|}{ Methyl tetradecanoate } \\
\hline 345.20 & 7.044 & 351.15 & 10.97 & 356.36 & 16.20 & & \\
\hline 348.06 & 8.683 & 353.20 & 12.69 & 359.13 & 19.75 & & \\
\hline \multicolumn{8}{|c|}{ Methyl pentadecanoate } \\
\hline 343.20 & 2.658 & 349.42 & 4.384 & 354.04 & 6.005 & 359.29 & 9.137 \\
\hline 345.38 & 3.215 & 351.83 & 5.254 & 355.35 & 6.843 & & \\
\hline 347.17 & 3.641 & 353.22 & 5.906 & 357.42 & 7.974 & & \\
\hline \multicolumn{8}{|c|}{ Methyl hexadecanoate } \\
\hline 344.18 & 1.429 & 351.99 & 2.500 & 353.88 & 3.160 & 358.04 & 4.107 \\
\hline 347.32 & 1.915 & 352.16 & 2.514 & 355.17 & 3.264 & 361.21 & 5.153 \\
\hline 349.23 & 2.157 & & & & & & \\
\hline
\end{tabular}


where $p$ is the saturation vapour pressure, $T$ the thermodynamic temperature, $\theta$ a chosen reference temperature and $p^{0}$ a reference pressure; in this work we take $p^{0}=1 \mathrm{~Pa}$. The operator $\Delta_{\text {vap }}$ stands for the value of the quantity in the vapour state minus the value of the same quantity in the liquid state, $G_{\mathrm{m}}^{\circ}$ is the molar Gibbs energy at $p=p^{0}, H_{\mathrm{m}}^{\circ}$ the molar enthalpy and $C_{p, \mathrm{~m}}^{\circ}$ is the molar heat capacity. The value of the gas constant $R$ is $8.314472 \mathrm{~J} \mathrm{~K}^{-1} \mathrm{~mol}^{-1}$. The use of Eq. (1) implies that apart from the assessed vapour pressures, the result of the regression procedure consists of the thermodynamic vaporisation quantities at the reference temperature and at $p=1 \mathrm{~Pa}$.

For a (pre-)screening of the data sets, to judge precision and mutual consistency, we make use of the 'arc method', which was proposed recently $[3,4]$. The arc method corresponds to a representation of data, in which the usual plot of $\ln p$ versus $1 / T$ is replaced by a plot of $\ln f$ versus $1 / T$. The function $\ln f$ is defined as

$$
\ln f=\ln p-\alpha+\frac{\beta}{T}
$$

In this expression, $\alpha$ and $\beta$ are constants that are fixed such that $\ln f$ is (practically) zero for the two extreme $(T, p)$ pairs of a given data set. The change from $\ln \left(p / p^{0}\right)$ to $\ln f$ corresponds to an amplification of the sensitivity of the ordinate axis by two orders of magnitude: details of the data that remain hidden in the traditional representation reveal themselves in the arc representation.

\section{Experimental data}

The experimental data by van Bommel [1] are given in Tables 1-3. The data in Table 1 were obtained by a static method, using an MKS baratron diaphragm manometer [5]. The data in Table 2 were obtained by simultaneous torsion and mass-loss effusion [6]. The data in Table 3 were obtained by means of the spinning rotor technique [7]. The experimental data by van Bommel are shown in Figs. 1-2, along with data from other sources. The latter are by Stage [8]; Rose and Supina [9]; Bonhorst et al. [10]; Jantzen and Witgert [11]; Rose et al. [12]; Scott et al. [13]; Husain et al. [14]; Spizzichino [15]; Norris and Terry [16]; Althouse and Triebold [17]; and Baccanari et al. [18].

Table 2

Vapour pressure data [1], torsion mass-loss effusion and temperatures (in K) for given pressure

\begin{tabular}{|c|c|c|c|c|c|c|c|c|c|c|}
\hline & \multicolumn{10}{|l|}{$p(\mathrm{~Pa})$} \\
\hline & 0.1 & 0.2 & 0.3 & 0.4 & 0.5 & 0.6 & 0.7 & 0.8 & 0.9 & 1.0 \\
\hline Methyl decanoate & 260.41 & 265.89 & 269.20 & 271.60 & 273.49 & 275.06 & 276.40 & 277.57 & 278.61 & 279.54 \\
\hline Methyl undecanoate & 271.35 & 276.95 & 280.33 & 282.78 & 284.71 & 286.31 & 287.67 & 288.87 & 289.93 & 290.88 \\
\hline Methyl dodecanoate & 281.68 & 287.48 & 290.98 & 293.52 & 295.52 & 297.17 & 298.59 & 299.82 & 300.92 & 301.91 \\
\hline Methyl tridecanoate & 291.53 & 297.46 & 301.03 & 303.62 & 305.66 & 307.35 & 308.79 & 310.05 & 311.17 & 312.18 \\
\hline Methyl tetradecanoate & 300.94 & 307.08 & 310.79 & 313.48 & 315.60 & 317.35 & 318.84 & 320.15 & 321.31 & 322.36 \\
\hline Methyl pentadecanoate & 310.17 & 316.45 & 320.24 & 322.98 & 325.14 & 326.93 & 328.45 & 329.79 & 330.97 & 332.04 \\
\hline Methyl hexadecanoate & 319.11 & 325.52 & 329.39 & 332.20 & 334.41 & 336.23 & 337.79 & 339.15 & 340.36 & 341.45 \\
\hline Methyl heptadecanoate & 327.60 & 334.15 & 338.11 & 340.98 & 343.23 & 345.10 & 346.69 & 348.08 & 349.32 & 350.43 \\
\hline Methyl octadecanoate & 335.95 & 342.60 & 346.62 & 349.53 & 351.81 & 353.71 & 355.32 & 356.73 & 357.99 & 359.12 \\
\hline Methyl nonadecanoate & 343.57 & 350.51 & 354.70 & 357.73 & 360.12 & 362.09 & 363.78 & 365.25 & 366.56 & 367.74 \\
\hline Methyl eicosonoate & 351.50 & 358.49 & 362.71 & 365.76 & 368.16 & 370.15 & 371.85 & 373.33 & 374.65 & 375.84 \\
\hline
\end{tabular}


Table 3

Vapour pressure data [1], and spinning rotor manometry

\begin{tabular}{|c|c|c|c|c|c|c|c|}
\hline$T(\mathrm{~K})$ & $p(\mathrm{mPa})$ & $T(\mathrm{~K})$ & $p(\mathrm{mPa})$ & $T(\mathrm{~K})$ & $p(\mathrm{mPa})$ & $T(\mathrm{~K})$ & $p(\mathrm{mPa})$ \\
\hline \multicolumn{8}{|c|}{ Methyl hexadecanoate } \\
\hline 303.46 & 14.66 & 303.54 & 14.10 & 305.75 & 19.48 & 310.89 & 35.84 \\
\hline 303.48 & 14.99 & 304.82 & 17.22 & 305.75 & 19.49 & 310.92 & 36.10 \\
\hline 303.48 & 16.30 & 304.82 & 17.36 & 308.67 & 27.58 & 312.88 & 44.67 \\
\hline \multicolumn{8}{|c|}{ Methyl heptadecanoate } \\
\hline 295.86 & 1.76 & 304.70 & 10.92 & 311.85 & 17.07 & 324.03 & 61.22 \\
\hline 299.85 & 3.48 & 304.74 & 8.57 & 318.06 & 32.41 & 324.06 & 62.45 \\
\hline \multicolumn{8}{|c|}{ Methyl octadecanoate } \\
\hline 316.04 & 9.96 & 322.99 & 22.05 & 321.66 & 19.85 & 323.94 & 25.59 \\
\hline
\end{tabular}

\section{Discussion}

\subsection{Screening of experimental data}

The arc representation of experimental vapour pressure data is a convenient, powerful tool for making a (pre-)screening of individual or combined data sets on a given substance.

The $\ln f$ function, underlying the arc representation and defined by Eq. (2), has the double advantage of being a physical representation and, at the same time, a representation which reveals details of the set(s) of data. As a physical representation, it distinguishes itself from the commonly used deviation plots. In contrast to the deviation plot, which is made after some mathematical treatment of the data, the $\ln f$ representation is equally useful before and after the mathematical treatment of the data.

The arc-like appearance of the $\ln f$ function, read the $\ln f$ representation of vapour pressure data, is the result of the influence of $\Delta_{\mathrm{vap}} C_{p, \mathrm{~m}}^{\circ}$, the difference in molar heat capacity of vapour and liquid. From the Clausius-Clapeyron equation

$$
\frac{\mathrm{d} \ln p}{\mathrm{~d}(1 / T)}=\frac{\Delta_{\mathrm{vap}} H_{\mathrm{m}}^{\circ}(T)}{R}
$$

it follows that the slope of the $\ln f$ function, Eq. (2), is given by

$$
\frac{\mathrm{d} \ln f}{\mathrm{~d}(1 / T)}=\frac{\Delta_{\mathrm{vap}} H_{\mathrm{m}}^{\circ}(T)}{R}+\beta
$$

The difference properties in Eq. (1) can be 'read' easily from the arc, from Eq. (1):

$$
\Delta_{\mathrm{vap}} G_{\mathrm{m}}^{\circ}\left(\text { at } T_{\max }\right)=-R T_{\max } \ln \left(\frac{p\left(T_{\max }\right)}{p^{0}}\right)
$$

from Eq. (4):

$$
\Delta_{\text {vap }} H_{\mathrm{m}}^{0}\left(\text { at } T_{\max }\right)=\beta R
$$


(a)

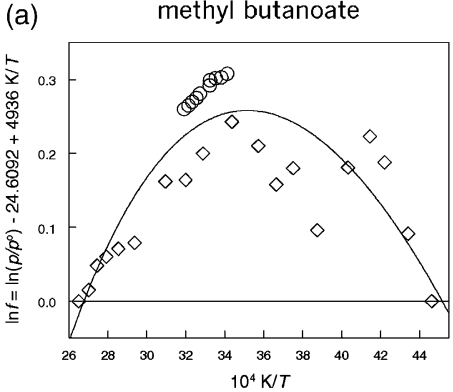

(d)

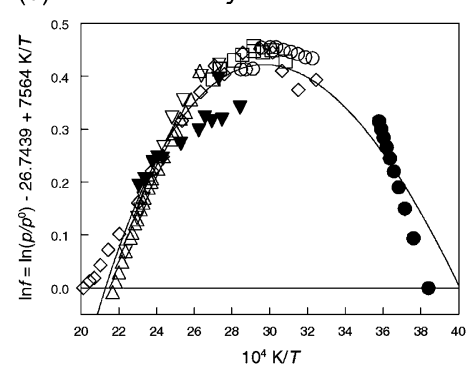

(g) methyl hexadecanoate

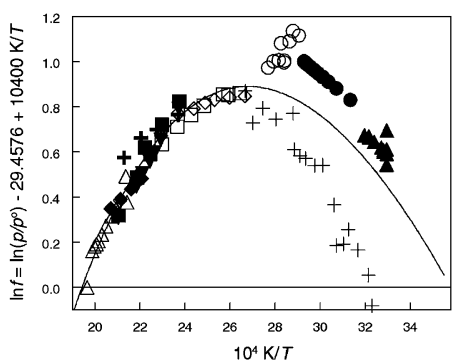

(b)

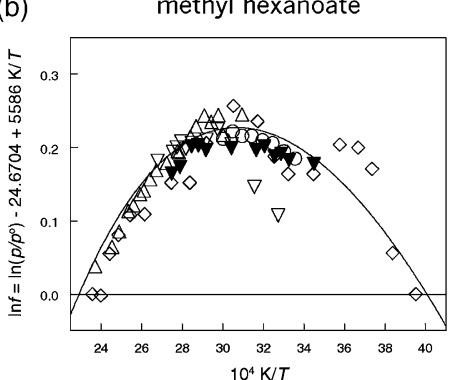

(e)

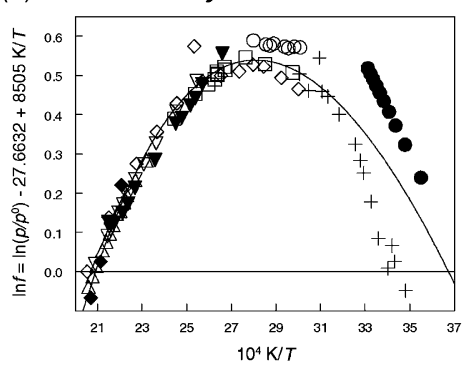

(h)

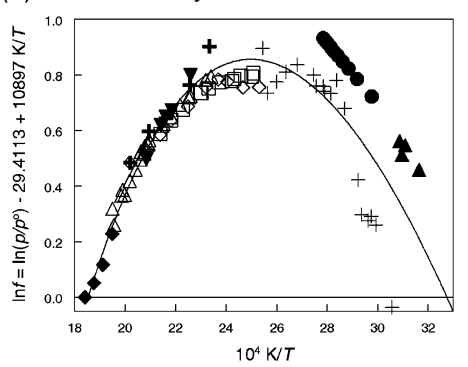

(c) methyl octanoate
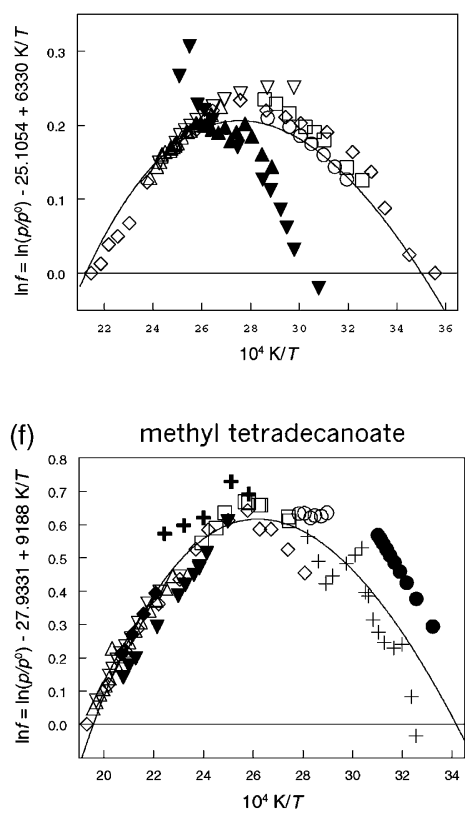

(i) methyl eicosonoate

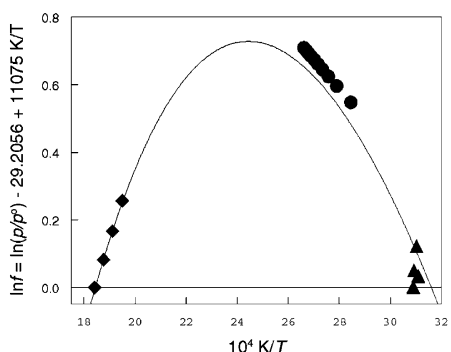

Fig. 1. (a-i) Vapour pressures for the methyl esters of the even carbonic acids, showing literature results and results obtained using the arc representation: $(\mathbf{O})$ [1] (effusion); $(\bigcirc)$ [1] (static); $(\mathbf{\Delta})$ [1] (spinning rotor); $(\triangle)[9] ;(\boldsymbol{\nabla})[10] ;(\nabla)[11] ;(\diamond)[8]$; $(\square)[13] ;(\diamond)[14] ;(\mathbf{+})[16] ;(\boldsymbol{\square})[17] ;(+)[15]$.

and by Taylor's series expansion [4]:

$$
\Delta_{\mathrm{vap}} C_{\mathrm{p}, \mathrm{m}}^{0} \cong-8 R\left(T_{\max } b\right)^{-2} h
$$

where $T_{\max }$ is the temperature corresponding to the maximum of the arc, $h$ the height of the arc, i.e. the $\ln f$ value at its maximum, and $b$ is the width of the arc at its base. From the difference properties at $T_{\max }$, the vapour pressures can be calculated at any temperature; by means of Eq. (1), and taking $\theta=T_{\max }$. In addition, from the difference properties at $T_{\max }$, the values of $\Delta_{\mathrm{vap}} H_{\mathrm{m}}^{\circ}$ and $\Delta_{\text {vap }} G_{\mathrm{m}}^{\circ}$ at $T=298.15 \mathrm{~K}$ are easily calculated. These observations imply that Eqs. (5)-(7) allow a reliable 'manual' treatment of a set of experimental data, i.e. without the use of least squares (for examples, see refs. [4,19]). 

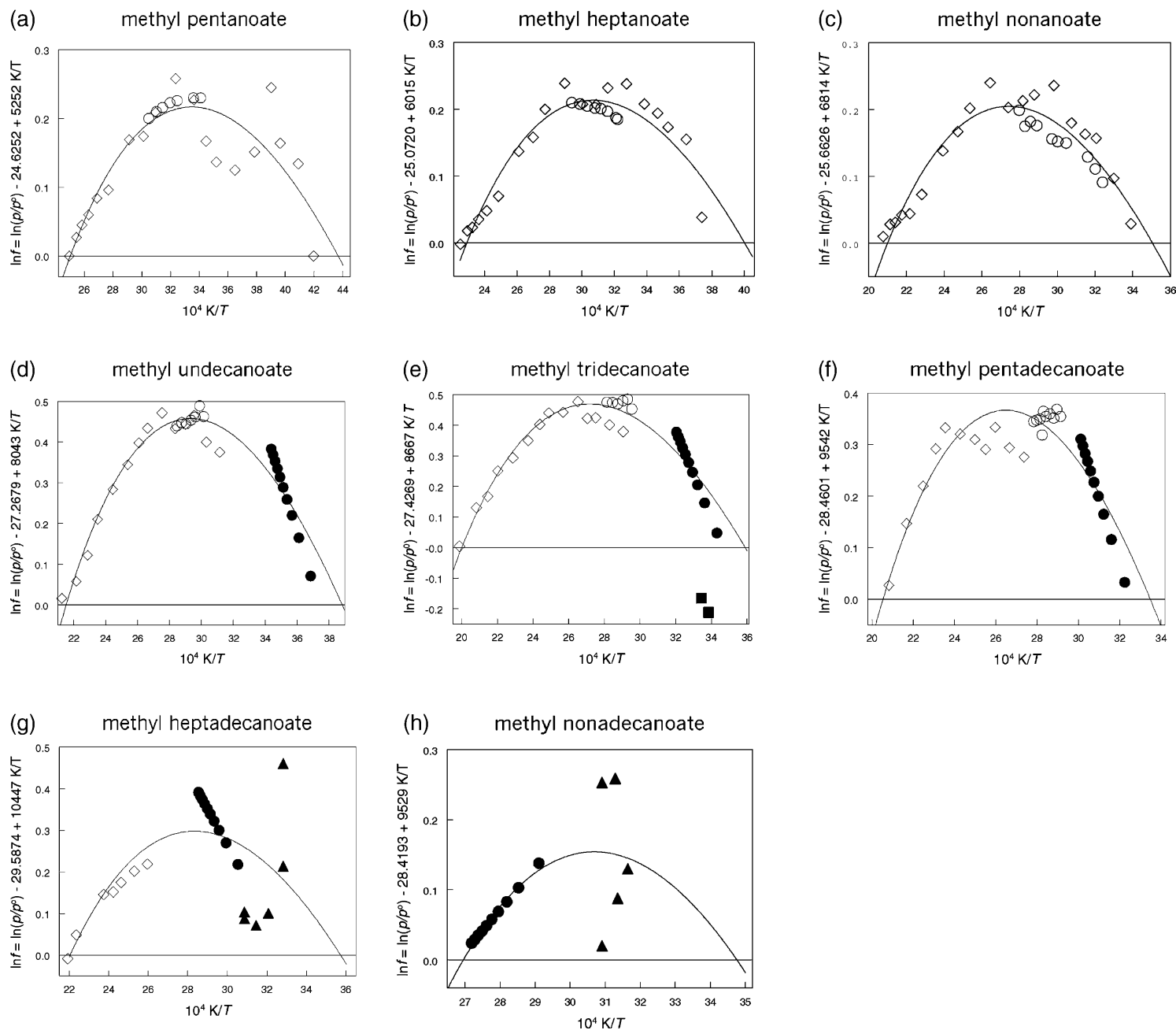

Fig. 2. (a-h) Vapour pressures for the methyl esters of the odd carbonic acids, showing literature results and results obtained

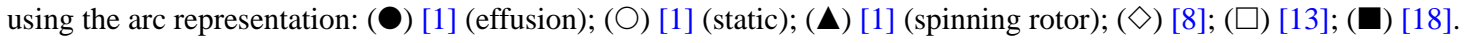

As an example of the use of Eq. (7) for the calculation of $\Delta_{\mathrm{vap}} C_{\mathrm{m}}^{\circ}$, we take the combined sets of data for methyl octanoate, Fig. 1c. From Fig. 1c, it follows that the maximum of the arc is at about $\left(10^{4} \mathrm{~K} / T_{\max }\right)=28$, or $T_{\max } \cong 357 \mathrm{~K}$. The height of the arc is about $h \cong 0.23$, and the width at its base (taking the open diamonds by Stage) $b=14 \times 10^{-4} \mathrm{~K}^{-1}$. With these values Eq. (7) yields the value of $\Delta_{\text {vap }} C_{p, \mathrm{~m}}^{\circ}=-61 \mathrm{~J} \mathrm{~K}^{-1} \mathrm{~mol}^{-1}$.

An important issue, when comparing sets of data, is pertaining to the quality (as regards accuracy and precision) of an individual set of data. As an example we take the set of static data by van Bommel for methyl octanoate; its $\ln f$ representation is shown in Fig. 3. Realising that a difference of 0.01 in $\ln f$ corresponds to a difference of $1 \%$ in pressure, one can observe that the data have a high precision (absence of random errors). The data also have a high degree of accuracy in that they are in close 


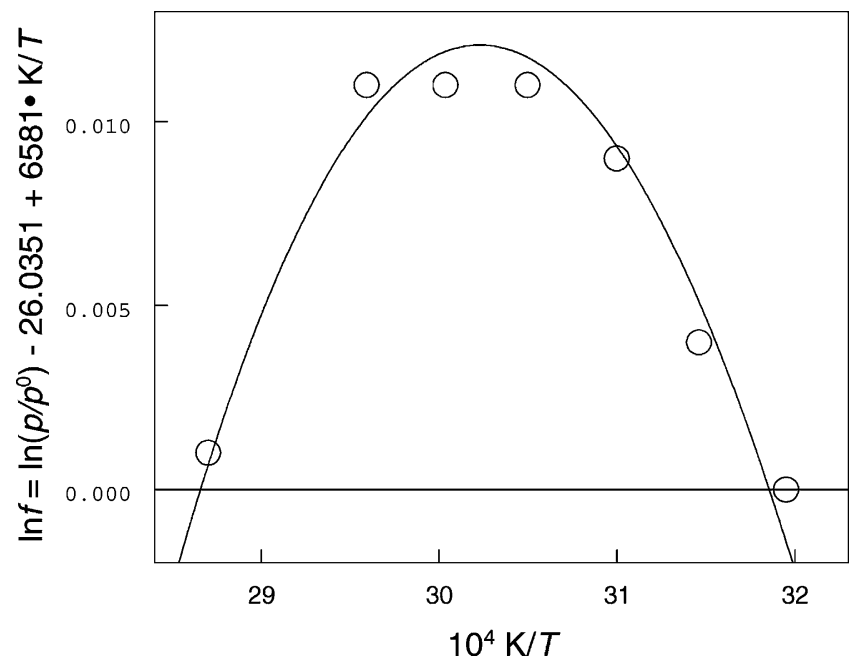

Fig. 3. Vapour pressures for methyl octanoate, showing literature results and results obtained using the arc representation: $(\bigcirc)$ [1] (static).

agreement with the data that set the overall trend. And, last but not least, Fig. 3 with Eq. (7) is giving rise to a $\Delta_{\mathrm{vap}} C_{\mathrm{m}}^{\circ}$ value of about $-60 \mathrm{~J} \mathrm{~K}^{-1} \mathrm{~mol}^{-1}$, i.e. in line with the value calculated from Fig. $1 \mathrm{c}$ (see earlier paragraph).

The situation sketched in the preceding paragraph is the exception rather than the rule. In most of the cases, an individual set over a limited range of temperature does not have the precision to reveal a 'reasonable' $\Delta_{\mathrm{vap}} C_{\mathrm{m}}^{\circ}$. As a matter of fact, the effusion instrument used by van Bommel does not yield any $\Delta_{\mathrm{vap}} C_{\mathrm{m}}^{\circ}$ at all. The instrument, designed by de Kruif et al. [5], is equipped with software which yields temperature values for evenly spaced pressures from 0.1 to $1.0 \mathrm{~Pa}$, and such that $\ln p$ is linear in $(1 / T)$.

The complete ensemble of experimental data, displayed by Figs. 1-2, allow us to make a number of additional observations.

1. For all of the substances, the combined data sets clearly display an arc-like behaviour. It implies that, for the mathematical processing of the data in terms of Eq. (1), three adjustable parameters $\left(\Delta_{\text {vap }} G_{\mathrm{m}}^{\circ}, \Delta_{\text {vap }} H_{\mathrm{m}}^{\circ}\right.$ and $\Delta_{\text {vap }} C_{p, \mathrm{~m}}^{\circ}$ ) are justified and necessary (the addition of the temperature derivative of $\Delta_{\mathrm{vap}} \Delta C_{p, \mathrm{~m}}^{\circ}$ ) as a fourth parameter is not justified: the data are not precise enough to reveal an asymmetric arc [20]).

2. Individual data sets, as a rule, differ from the average trend, in a systematic sense. Systematic differences between data from different sources are most noticeable at the lowest pressures (because of the nature of the presentation and the fact that the measurement of low pressures makes high demands). As an example, van Bommel's effusion (range 0.1-1.0 Pa) and spinning rotor data (0.001-0.1 Pa) are systematically higher in pressure than Spizzichino's effusion data (0.013-13 Pa), see Fig. 2a-d.

3. For a given source/laboratory (eventually in combination with a given technique), the quality of the data sets (consistency with average trend, and precision) differs from substance to substance, in a rather random manner. For example, the data by Bonhorst are in line with the data from other sources, however, with the striking exception of methyl octanoate. 


\subsection{Thermodynamic vaporisation properties}

Mathematical processing of the experimental data in terms of Eq. (1) is yielding the numerical values of the vaporisation quantities $\Delta_{\mathrm{vap}} G_{\mathrm{m}}^{\circ}(\theta), \Delta_{\mathrm{vap}} H_{\mathrm{m}}^{\circ}(\theta)$, and $\Delta_{\mathrm{vap}} C_{p, \mathrm{~m}}^{\circ}$. The computed value of $\Delta_{\mathrm{vap}} C_{p, \mathrm{~m}}^{\circ}$ is independent of the choice of $\theta$, whereas the computed values of $\Delta_{\text {vap }} G_{\mathrm{m}}^{\circ}$ and $\Delta_{\mathrm{vap}} H_{\mathrm{m}}^{\circ}$ depend on the choice of $\theta$. In other terms, there is only one mathematical result, in which the choice of $\theta$ is affecting the values computed for $\Delta_{\mathrm{vap}} G_{\mathrm{m}}^{\circ}$ and $\Delta_{\mathrm{vap}} H_{\mathrm{m}}^{\circ}$.

As a first step, we took $\theta=350 \mathrm{~K}$ for all of the substances, including all data sets and giving them equal weight. The result of the computations is part of Table 4; the numerical values are given as integers, passing over the question of the number of significant digits. The computed $\Delta_{\mathrm{vap}} G_{\mathrm{m}}^{\circ}$ and $\Delta_{\mathrm{vap}} H_{\mathrm{m}}^{\circ}$ values are plotted in Fig. 2, as a function of the number of carbon atoms in the molecules of the parent acids. Similarly, the computed $\Delta_{\mathrm{vap}} C_{p, \mathrm{~m}}^{\circ}$ values are displayed in Fig. 5.

Out of the three vaporisation quantities, $\Delta_{\mathrm{vap}} G_{\mathrm{m}}^{\circ}$ is the one which is most directly related to the measured vapour pressures. Therefore, it follows from Fig. 4 that the overall consistency, between the results for the different substances, is satisfactory. From the same figure, it follows that the dispersion of the $\Delta_{\mathrm{vap}} H_{\mathrm{m}}^{\circ}$ values is higher, the main cause being the inaccuracy of (some of) the computed $\Delta_{\mathrm{vap}} C_{p, \mathrm{~m}}^{\circ}$ values (see Fig. 5).

In order to arrive at a collection of vaporisation properties for $T=298.15 \mathrm{~K}$, we started from two (rather obvious) assumptions. In the first place, it is not implausible to assume that, for a given substance, the physical significance of the computed $\Delta_{\mathrm{vap}} G_{\mathrm{m}}^{\circ}$ and $\Delta_{\mathrm{vap}} H_{\mathrm{m}}^{\circ}$ values is maximal at the top of the arc, or rather at the temperature corresponding to the top. To bring the top values of $\Delta_{\mathrm{vap}} G_{\mathrm{m}}^{\circ}$ and $\Delta_{\mathrm{vap}} H^{\circ}$ to their values at $T=298.15 \mathrm{~K}$, the values of $\Delta_{\text {vap }} C_{p, \mathrm{~m}}^{\circ}$ are needed. Our second assumption is that more reliable results are obtained by using a calculated $\Delta_{\text {vap }} C_{p, \mathrm{~m}}^{\circ}$ (from a fit of all of the individual $\Delta_{\text {vap }} C_{p, \mathrm{~m}}^{\circ}$ values in column 4 of Table 4 as a function of number of carbon atoms) than by using the individually calculated $\Delta_{\mathrm{vap}} C_{p, \mathrm{~m}}^{\circ}$ values in column 4 of Table 4 .

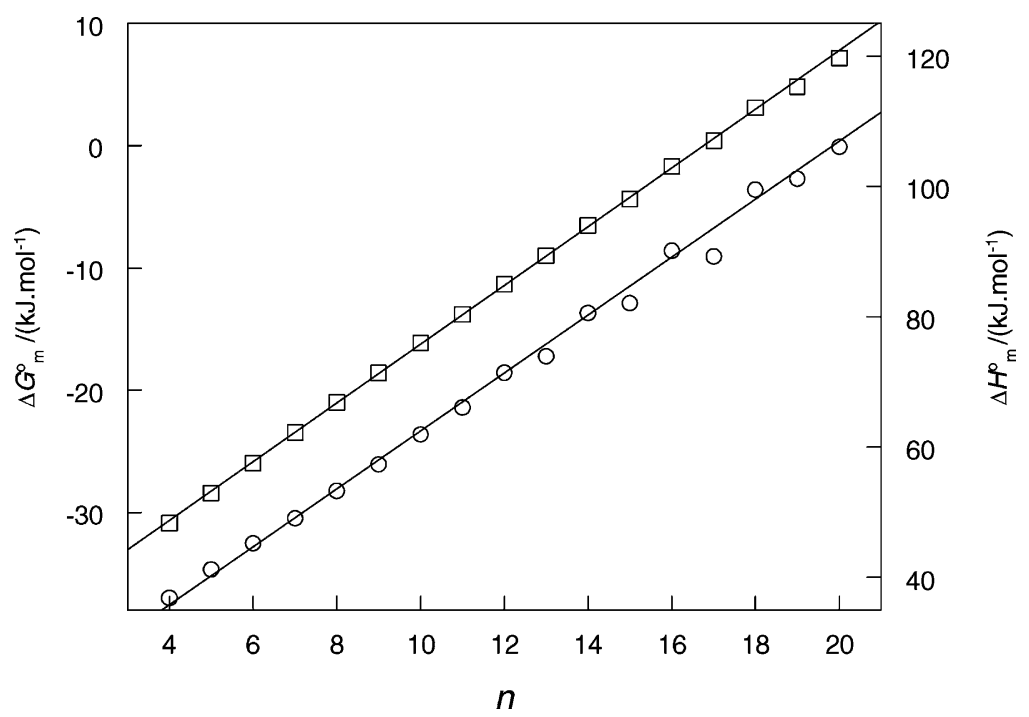

Fig. 4. Computed $\Delta_{\text {vap }} G_{\mathrm{m}}^{\circ}(\square)$ and $\Delta_{\text {vap }} H_{\mathrm{m}}^{\circ}(\bigcirc)$, respectively as a function of the number of carbon atoms of the parent acids at $\theta=350 \mathrm{~K}$. 
Table 4

Survey of results of computations

\begin{tabular}{|c|c|c|c|c|c|c|c|c|c|c|}
\hline$n$ & $\begin{array}{l}\Delta G_{\mathrm{m}}^{\circ} \\
\left(\mathrm{J} \mathrm{mol}^{-1}\right)\end{array}$ & $\begin{array}{l}\Delta H_{\mathrm{m}}^{\circ} \\
\left(\mathrm{J} \mathrm{mol}^{-1}\right)\end{array}$ & $\begin{array}{l}\Delta C_{p, \mathrm{~m}}^{\circ} \\
\left(\mathrm{J} \mathrm{K}^{-1} \mathrm{~mol}^{-1}\right)\end{array}$ & $S$ & $\theta(\mathrm{K})$ & $\begin{array}{l}\Delta G_{\mathrm{m}}^{\circ} \\
\left(\mathrm{J} \mathrm{mol}^{-1}\right)\end{array}$ & $\begin{array}{l}\Delta H_{\mathrm{m}}^{\circ} \\
\left(\mathrm{J} \mathrm{mol}^{-1}\right)\end{array}$ & $\begin{array}{l}\Delta C_{p, \mathrm{~m}}^{\circ} \\
\left(\mathrm{J} \mathrm{K}^{-1} \mathrm{~mol}^{-1}\right)\end{array}$ & $\begin{array}{l}\Delta G_{\mathrm{m}}^{\circ} \\
\left(\mathrm{kJ} \mathrm{mol}^{-1}\right)\end{array}$ & $\begin{array}{l}\Delta H_{\mathrm{m}}^{\circ} \\
\left(\mathrm{kJ} \mathrm{mol}^{-1}\right)\end{array}$ \\
\hline & $\theta=350 \mathrm{~K}$ & & & & & & & $T=298.15 \mathrm{~K}$ & & \\
\hline 4 & -30883 & 36890 & -63.2 & 0.053 & 284 & $-17683 \pm 33$ & $41060 \pm 172$ & -30 & $-20.60 \pm 0.03$ & $40.64 \pm 0.22$ \\
\hline 5 & -28428 & 41272 & -46.7 & 0.038 & 299 & $-18090 \pm 26$ & $43653 \pm 136$ & -39 & $-17.91 \pm 0.03$ & $43.69 \pm 0.14$ \\
\hline 6 & -25971 & 45188 & -49.9 & 0.027 & 325 & $-20842 \pm 13$ & $46437 \pm 57$ & -48 & $-15.23 \pm 0.01$ & $47.71 \pm 0.10$ \\
\hline 7 & -23489 & 49131 & -45.9 & 0.023 & 326 & $-18470 \pm 17$ & $50232 \pm 112$ & -56 & $-12.53 \pm 0.02$ & $51.80 \pm 0.13$ \\
\hline 8 & -21014 & 53341 & -55.1 & 0.042 & 363 & $-23763 \pm 17$ & $52625 \pm 125$ & -65 & $-9.71 \pm 0.02$ & $56.87 \pm 0.14$ \\
\hline 9 & -18602 & 57416 & -51.7 & 0.068 & 364 & $-21629 \pm 58$ & $56692 \pm 299$ & -74 & $-6.99 \pm 0.07$ & $61.59 \pm 0.39$ \\
\hline 10 & -16145 & 61952 & -70.6 & 0.040 & 337 & $-13227 \pm 53$ & $62869 \pm 87$ & -83 & $-4.26 \pm 0.05$ & $66.10 \pm 0.17$ \\
\hline 11 & -13799 & 66079 & -88.0 & 0.037 & 340 & $-11504 \pm 26$ & $66959 \pm 127$ & -92 & $-1.60 \pm 0.05$ & $70.82 \pm 0.35$ \\
\hline 12 & -11324 & 71421 & -113.5 & 0.080 & 356 & $-12737 \pm 81$ & $70740 \pm 166$ & -101 & $1.33 \pm 0.10$ & $76.59 \pm 0.41$ \\
\hline 13 & -8987 & 73955 & -91.8 & 0.011 & 368 & $-13211 \pm 34$ & $72302 \pm 150$ & -110 & $3.80 \pm 0.07$ & $79.99 \pm 0.49$ \\
\hline 14 & -6534 & 79828 & -120.7 & 0.085 & 382 & $-14259 \pm 76$ & $75966 \pm 178$ & -119 & $6.73 \pm 0.15$ & $85.94 \pm 0.76$ \\
\hline 15 & -4346 & 82100 & -102.4 & 0.041 & 372 & $-9710 \pm 345$ & $79846 \pm 209$ & -128 & $9.07 \pm 0.40$ & $89.29 \pm 0.79$ \\
\hline 16 & -1657 & 93363 & -213.7 & 0.168 & 397 & $-13771 \pm 101$ & $83320 \pm 405$ & -137 & $12.25 \pm 0.22$ & $96.84 \pm 0.63$ \\
\hline 17 & 381 & 89299 & -85.2 & 0.103 & 353 & $-380 \pm 91$ & $89043 \pm 701$ & -146 & $14.17 \pm 0.13$ & $97.03 \pm 1.23$ \\
\hline 18 & 3011 & 98021 & -157.9 & 0.113 & 401 & $-10273 \pm 79$ & $89968 \pm 280$ & -155 & $17.68 \pm 0.23$ & $105.87 \pm 1.37$ \\
\hline 19 & 4827 & 101170 & -159.0 & 0.060 & 326 & $11567 \pm 71$ & $104980 \pm 2400$ & -164 & $19.75 \pm 0.08$ & $109.53 \pm 2.72$ \\
\hline 20 & 7308 & 109200 & -203.0 & 0.045 & 406 & $-8131 \pm 66$ & $97836 \pm 204$ & -172 & $22.74 \pm 0.27$ & $116.43 \pm 1.54$ \\
\hline
\end{tabular}

Column 1: number of carbon atoms in the molecules of the parent alkanoic acid. Columns $2-4$ : result of data processing in terms of Eq. (1), taking $\theta=350 \mathrm{~K}$. Column 5: computed S.D. Columns 6-8: result of data processing in terms of Eq. (1), taking for $\theta$ the temperature corresponding to the maximum of the arc. Column 9: smoothed heat capacity difference. Columns 10 and 11: calculated standard Gibbs energy and enthalpy of vaporisation, respectively and valid for $T=298.15 \mathrm{~K}$. 


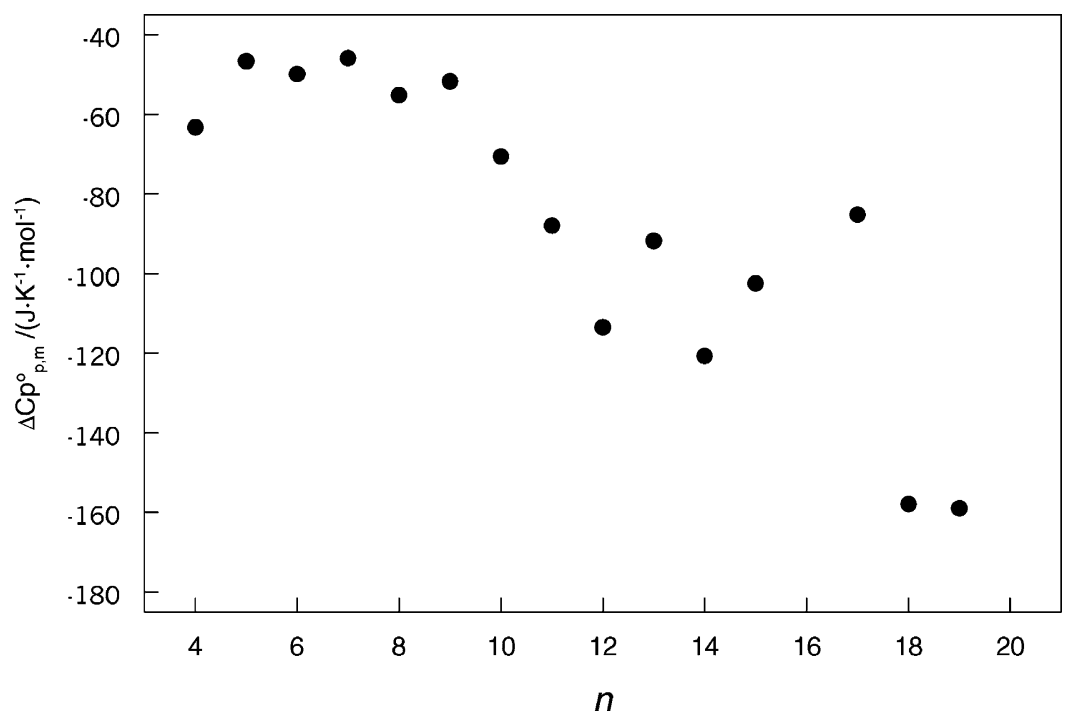

Fig. 5. Computed $\Delta_{\mathrm{vap}} C_{p, \mathrm{~m}}^{\circ}$ as a function of the number of carbon atoms of the parent acids at $\theta=350 \mathrm{~K}$.

The $\Delta_{\text {vap }} G_{\mathrm{m}}^{\circ}$ and $\Delta_{\mathrm{vap}} H_{\mathrm{m}}^{\circ}$, calculated for the individual substances with their own $\theta$, are included in Table 4 along with their mathematically computed standard deviations. The $\Delta_{\mathrm{vap}} C_{p, \mathrm{~m}}^{\circ}$ values needed to transfer the $\Delta_{\mathrm{vap}} G_{\mathrm{m}}^{\circ}$ and $\Delta_{\mathrm{vap}} H_{\mathrm{m}}^{\circ}$ to 298.15 , satisfy the equation

$$
\Delta_{\mathrm{vap}} C_{p, m}^{\circ}(n)=5.64-8.929 n
$$

and are included in column 9 of Table 4.

The calculated $\Delta_{\text {vap }} H_{\mathrm{m}}^{\circ}($ at $T=298.15 \mathrm{~K})$ and $\Delta_{\mathrm{vap}} G_{\mathrm{m}}^{\circ}($ at $T=298.15 \mathrm{~K})$ are given in Table 4 , and graphically represented by Fig. 6 . The data in Table 4 are provided with estimated uncertainties that reflect the standard deviations of the least-squares calculation along with the uncertainties introduced by the extrapolation from $T=\theta$ to $298.15 \mathrm{~K}$.

Finally, we subjected the data for $T=298.15 \mathrm{~K}$ to a linear least-squares treatment, such that they were provided with weight factors. These factors were taken inversely proportional to the estimated uncertainties displayed in Table 4.

The final result is represented by the following two equations:

$$
\begin{gathered}
\Delta_{\text {vap }} G_{\mathrm{m}}^{\circ}(\text { at } T=298.15 K, n)=-31.39+2.708 n \\
\Delta_{\text {vap }} H_{\mathrm{m}}^{\circ}(\text { at } T=298.15 \mathrm{~K}, n)=19.39+4.741 n
\end{gathered}
$$

In our view, the last two equations are the most accurate representations of the two thermodynamic vaporisation quantities that can be derived from the complete amount of experimental vapour pressure data. The precisions of the numerical values, calculated by the Eqs. (9) and (10), are a function of $n$, reaching their lowest values for $n=6$, i.e. for methyl hexanoate. We estimate that the uncertainties $(\delta)$ in $\Delta_{\mathrm{vap}} G_{\mathrm{m}}^{\circ}$ are given by

$$
\delta\left(\Delta_{\mathrm{vap}} G_{\mathrm{m}}^{\circ}, n\right)=\{0.01+0.015(|n-6|)\}
$$




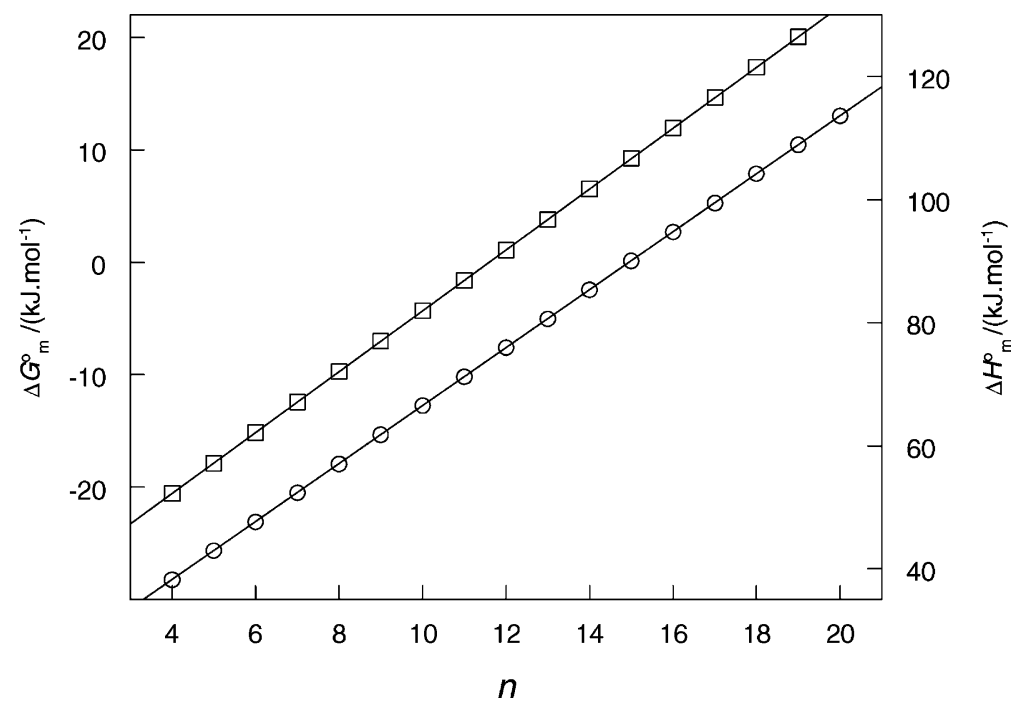

Fig. 6. Computed $\Delta_{\text {vap }} G_{\mathrm{m}}^{\circ}(\square)$ and $\Delta_{\text {vap }} H_{\mathrm{m}}^{\circ}(\bigcirc)$, respectively as a function of the number of carbon atoms of the parent acids at $T=298.15 \mathrm{~K}$.

and those in $\Delta_{\text {vap }} H_{\mathrm{m}}^{\circ}$ by

$$
\delta\left(\Delta_{\mathrm{vap}} H_{\mathrm{m}}^{\circ}, n\right)=\{0.10+0.053(|n-6|)\}
$$

\section{Conclusions}

- Vapour pressure data on the methyl esters of the normal alkanoic acids, $\mathrm{C}_{n} \mathrm{H}_{2 n-1} \mathrm{O}_{2} \mathrm{CH}_{3}$, for $4 \leq n \leq$ 20 , from the sources available in literature, have been combined and mathematically processed in terms of Eq. (1).

- The computational result, for the chosen reference temperature of $\theta=350 \mathrm{~K}$, is represented by the columns 2-5 of Table 4. Column 5 gives the calculated standard deviation between experimental and calculated vapour pressure in a relative sense.

- The physical significance of the computed vaporisation quantities $\Delta_{\mathrm{vap}} G_{\mathrm{m}}^{\circ}$ and $\Delta_{\mathrm{vap}} H_{\mathrm{m}}^{\circ}$, for each of the substances, is maximal at the temperature corresponding to the maximum of the arc representation. The relevant information, i.e. $T_{\max }, \Delta_{\mathrm{vap}} G_{\mathrm{m}}^{\circ}\left(\right.$ at $\left.T_{\max }\right), \Delta_{\mathrm{vap}} H_{\mathrm{m}}^{\circ}\left(\right.$ at $T_{\max }$ ), is represented by the columns 6-8 of Table 4.

- The numerical information contained in columns 6-8 of Table 4, along with the smoothed $\Delta_{\text {vap }} \Delta C_{p, \mathrm{~m}}^{\circ}$ values, from Eq. (8) and given in column 9 of Table 4, has been used to calculate for $T=298.15 \mathrm{~K}$, the $\Delta_{\text {vap }} G_{\mathrm{m}}^{\circ}$ and $\Delta_{\text {vap }} H_{\mathrm{m}}^{\circ}$ values in columns 10 and 11 in Table 4 . The uncertainties in the $\Delta_{\text {vap }} G_{\mathrm{m}}^{\circ}$ and $\Delta_{\text {vap }} H_{\mathrm{m}}^{\circ}$ values, in columns 10 and 11 of Table 4 , are estimated uncertainties.

- As a function of the number $(n)$ of carbon atoms in the molecules of the parent acids, the overall result of the investigation, valid for $T=298.15 \mathrm{~K}$, is represented by Eq. (9) for $\Delta_{\mathrm{vap}} G_{\mathrm{m}}^{\circ}$ and Eq. (10) for $\Delta_{\text {vap }} H_{\mathrm{m}}^{\circ}$. 


\section{References}

[1] M.J. van Bommel, Thermodynamic Behaviour of Methyl Esters of Long Chain Linear Carboxylic Acids, Thesis, Utrecht University, Utrecht, The Netherlands, 1986.

[2] E.C.W. Clarke, D.N. Glew, Trans. Faraday Soc. 62 (1966) 539-547.

[3] H.A.J. Oonk, P.R. van der Linde, J. Huinink, J.G. Blok, J. Chem. Thermodyn. 30 (1998) 897-907.

[4] P.R. van der Linde, J.G. Blok, H.A.J. Oonk, J. Chem. Thermodyn. 30 (1998) 909-917.

[5] C.G. de Kruif, T. Kuipers, J.C. van Miltenburg, R.C.F. Schaake, G. Stevens, J. Chem. Thermodyn. 13 (1981) $1081-1086$.

[6] C.G. de Kruif, C.H.D. van Ginkel, J. Chem. Thermodyn. 9 (1977) 725-730.

[7] P.J. van Ekeren, M.H.G. Jacobs, J.C.A. Offringa, C.G. de Kruif, J. Chem. Thermodyn. 15 (1983) $409-417$.

[8] H. Stage, Fette und Seifen 55 (1953) 217-224.

[9] A. Rose, W.R. Supina, J. Chem. Eng. Data 6 (1961) 173-179.

[10] C.W. Bonhorst, P.M. Althouse, H.O. Triebold, Ind. Eng. Chem. 40 (1948) 2379-2384.

[11] E. Jantzen, H. Witgert, Fette und Seifen 46 (1939) 563-569.

[12] A. Rose, J.A. Acciarri, R.C. Johnson, W.W. Sanders, Ind. Eng. Chem. 49 (1957) 104-109.

[13] T.A. Scott, D. Macmillan, E.H. Melvin, Ind. Eng. Chem. 44 (1952) 172-175.

[14] S. Husain, P.N. Sarma, G.Y.S.K. Swamy, K.S. Devi, J. Am. Oil Chem. Soc. 70 (1993) 149-155.

[15] C. Spizzichino, J. Rech. CNRS 34 (1956) 1-24.

[16] F.A. Norris, D.E. Terry, Oil \& Soap 22 (1945) 41-46.

[17] P.M. Althouse, H.O. Triebold, Ind. Eng. Chem. Anal. Ed. 16 (1944) 605-606.

[18] D.P. Baccanari, J.A. Novinski, Y.-C. Pan, M.M. Yevitz, H.A. Swain Jr., Trans. Faraday Soc. 64 (1968) 1201-1205.

[19] H.A.J. Oonk, A.C.G. van Genderen, J.G. Blok, P.R. van der Linde, Phys. Chem. Chem. Phys. 2 (2000) 5614-5618.

[20] J.G. Blok, A.C.G. van Genderen, P.R. van der Linde, H.A.J. Oonk, J. Chem. Thermodyn. 33 (2001) 1097-1106. 